\title{
Synthesis and Antimicrobial Studies of Some Novel Pyrazoline and Isoxazoline Derivatives
}

\author{
S. B. JADHAV*, R. A. SHASTRI, K. V. GAIKWAD and S. V. GAIKWAD \\ P. G. Department of Chemistry, \\ Milind College of Science, Aurangabad-431001, Maharashtra, India. \\ orgchem.jadhav@yahoo.co.in
}

Received 5 April 2009; Accepted 1 June 2009

\begin{abstract}
A new series of 1H-3-(4'-substituted phenyl)-5-(6' '-methoxy napthaline)-2-pyrazolines (4a-e) and 1H-3-(4'-substituted phenyl)-5-(6', methoxynapthaline)-2-isoxazolines (5a-e) were synthesized by reacting 1-(4'-substituted phenyl)-3-(6''-methoxynapthaline)-2-propene-1-one (3a-e) with hydrazine hydrate and hydroxylamine hydrochloride respectively. All these compounds were characterized by means of their IR, ${ }^{1} \mathrm{H}$ NMR, spectroscopic data and microanalysis. All the synthesized products were evaluated for their antimicrobial activity. All the compounds exhibited significant to moderate antimicrobial activity.
\end{abstract}

Keywords: Chalcones, 2-Pyrazolines, 2-Isoxazolines, Antimicrobial activity.

\section{Introduction}

Heterocyclic compounds have so far been synthesized mainly due to the wide range of biological activities. Much attention has paid to the synthesis of heterocyclic compounds bearing nitrogen and oxygen containing ring system, like pyrazoline and isoxazoline mainly due to their higher pharmacological activity.

Pyrazoline are prominent nitrogen containing heterocyclic compounds play important role in medicinal chemistry. Considerable attention has been focused on pyrazoline derivatives due to their interesting biological activities. They have found to possess antifungal $^{1}$, antibacterial ${ }^{2}$, antidepressant ${ }^{3}$, anticonvulsant ${ }^{4,5}$, anti-inflammatory ${ }^{6}$, anti-tumar ${ }^{7}$, antidiabetic, anaesthetic and analgesic ${ }^{8-10}$ properties. Owing to the mentioned biological activities of pyrazoline prompted us to synthesize various substituted pyrazoline derivatives. Synthesis of novel isoxazoline derivative remains a main focus of medicinal chemist, due to their diverse pharmacological activity. Isoxazoline derivatives have been reported to possess antifungal $^{11,12}$, antibacterial ${ }^{13}$, anticonvulsant ${ }^{14}$, anti-inflammatory ${ }^{15}$, antiviral ${ }^{16}$ and analgesic ${ }^{17}$ activity. In addition, isoxazoline derivatives have played a crucial role as intermediates in the organic synthesis of number of heterocyclic pharmacological active compounds. 
Encouraged by the diverse biological activities of pyrazoline and isoxazoline compounds, it was decided to prepare a new series of pyrazoline and isoxazoline derivatives. These derivatives contains naphthalene moiety which is also substituted by methoxy group. Literature survey revealed that incorporation of naphthalene and halogen moiety in pyrazoline and isoxazoline ring enhanced antibacterial and antifungal activity. In the present communication, chalcones (3a-e) were prepared by the action of substituted acetophenone (1a-e) with 6-methoxy napthaldehyde in the presence of aqueous solution of potassium hydroxide and methanol at room temperature by Claisen-Schmidt condensation method. The synthesized chalcones further condensed with hydrazinehydrate in neutral media to give pyrazoline derivatives (4a-e). Similarly prepared chalcones reacted with hydroxyl amine hydrochloride in presence of pyridine to obtained isoxazoline derivatives (5a-e) Scheme 1. The structures of all synthesized compounds were assigned on the basis of IR, Mass, ${ }^{1} \mathrm{H}$ NMR spectral data and elemental analysis. Further these compounds were subjected for antifungal and antibacterial activity.

\section{Experimental}

Melting points (m.p.) were determined in open capillary tube and are uncorrected. IR spectra were recorded using a Perkin-Elmer 1600 FT spectrometer at a ca. $5-15 \%$ solution in DMSO- $\mathrm{d}_{6}$ or $\mathrm{CDCl}_{3}$ (TMS as internal standard). Thin layer chromatography (TLC) was performed on silica gel $\mathrm{G}$ for TLC (Merck) and spots were visualized by iodine vapors or by irradiation with ultraviolet lights $(254 \mathrm{~nm})$. Physical constants and analytical data of all the compounds reported in this paper are summarized in Table 1.

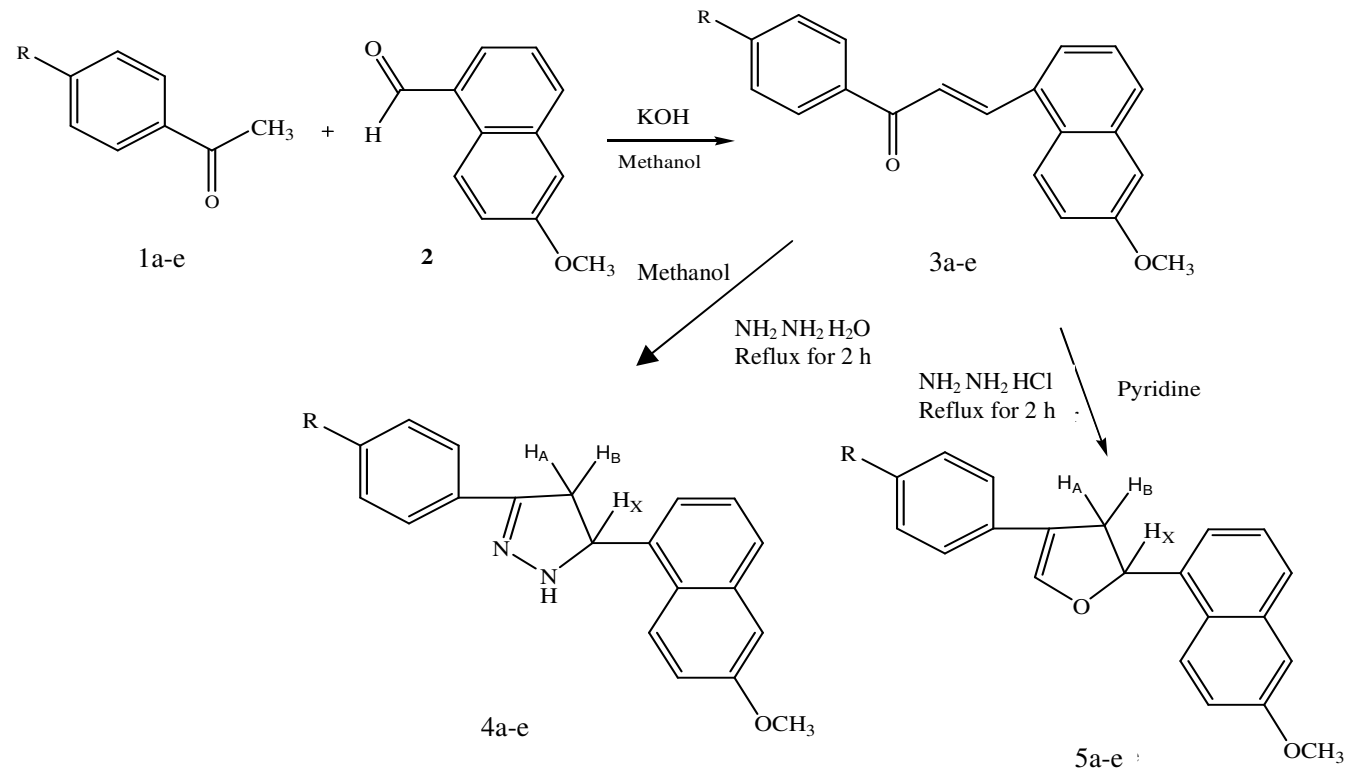

Scheme 1

General procedure for the synthesis of chalcones (3a-e)

A mixture of substituted acetophenones ( 0.01 mole) and 6-methoxy napthaldehyde $(0.01$ mole) was stirred in methanol $(50 \mathrm{~mL})$ and then a solution of $15 \mathrm{~mL}$ potassium hydroxide ( 0.02 mole) was added to it. The mixture was kept over night at room temperature and then it was poured into crushed ice and acidified with dil. $\mathrm{HCl}$. The chalcones derivative precipitates out as solid. Then it was filtered and crystallized from acetic acid Scheme 1. 


\section{General procedure for the synthesis of 2-pyrazoline (4a-e)}

A mixture of chalcones $(0.01$ mole $)$ and hydrazine hydrate $(0.02$ mole $)$ in $50 \mathrm{~mL}$ methanol was reflux for $2 \mathrm{~h}$, excess methanol was distilled and the resulting solution was kept overnight. Crystalline solid filtered and crystallized from ethanol Scheme I. Physical and analytical data of compounds are given in Table 1.

Table 1. Physical and analytical data of compounds (4a-e, 5a-e).

\begin{tabular}{cccccccc}
\hline $\begin{array}{c}\text { Compd. } \\
\text { No. }\end{array}$ & $\mathrm{R}$ & $\mathrm{M} . \mathrm{F}$ & $\begin{array}{c}\mathrm{m} . \mathrm{p} \\
0\end{array}$ & $\begin{array}{c}\text { Yield } \\
\%\end{array}$ & \multicolumn{3}{c}{$\begin{array}{c}\text { Analysis } \\
\text { Found (calcd) }\end{array}$} \\
\hline $\mathbf{4 a}$ & $\mathrm{H}$ & $\mathrm{C}_{20} \mathrm{H}_{18} \mathrm{~N}_{2} \mathrm{O}$ & 146 & 85 & $79.30(79.44)$ & $5.75(6.00)$ & $9.00(9.26)$ \\
$\mathbf{4 b}$ & $\mathrm{Br}$ & $\mathrm{C}_{20} \mathrm{H}_{17} \mathrm{BrN}_{2} \mathrm{O}$ & 136 & 80 & $62.75(63.00)$ & $4.29(4.29)$ & $7.15(7.35)$ \\
$\mathbf{4 c}$ & $\mathrm{F}$ & $\mathrm{C}_{20} \mathrm{H}_{17} \mathrm{FN}_{2} \mathrm{O}$ & 180 & 90 & $74.50(74.98)$ & $5.15(5.35)$ & $8.65(8.74)$ \\
$\mathbf{4 d}$ & $\mathrm{CH}_{3}$ & $\mathrm{C}_{21} \mathrm{H}_{20} \mathrm{~N}_{2} \mathrm{O}$ & 125 & 85 & $79.62(79.72)$ & $6.17(6.37)$ & $8.55(8.85)$ \\
$\mathbf{4 e}$ & $\mathrm{OCH}_{3}$ & $\mathrm{C}_{21} \mathrm{H}_{20} \mathrm{~N}_{2} \mathrm{O}_{2}$ & 165 & 70 & $75.55(75.88)$ & $5.95(6.06)$ & $8.23(8.43)$ \\
$\mathbf{5 a}$ & $\mathrm{H}$ & $\mathrm{C}_{20} \mathrm{H}_{17} \mathrm{NO}_{2}$ & 168 & 80 & $75.60(75.88)$ & $5.86(6.06)$ & $8.31(8.43)$ \\
$\mathbf{5 b}$ & $\mathrm{Br}$ & $\mathrm{C}_{20} \mathrm{H}_{16} \mathrm{BrNO}_{2}$ & 178 & 92 & $75.55(75.88)$ & $5.96(6.06)$ & $8.25(8.43)$ \\
$\mathbf{5 c}$ & $\mathrm{F}$ & $\mathrm{C}_{20} \mathrm{H}_{16} \mathrm{FNO}_{2}$ & 135 & 95 & $74.45(74.75)$ & $4.72(5.02)$ & $4.15(4.36)$ \\
$\mathbf{5 d}$ & $\mathrm{CH}$ & $\mathrm{C}_{21} \mathrm{H}_{19} \mathrm{NO}_{2}$ & 165 & 80 & $79.32(79.47)$ & $5.85(6.03)$ & $4.28(4.41)$ \\
$\mathbf{5 e}$ & $\mathrm{OCH}_{3}$ & $\mathrm{C}_{21} \mathrm{H}_{19} \mathrm{NO}_{3}$ & 150 & 75 & $75.35(75.66)$ & $5.52(5.74)$ & $3.95(4.20)$ \\
\hline
\end{tabular}

General procedure for the synthesis of 2-Isoxazoline (5a-e)

A mixture of chalcones $(0.01$ mole $)$ and hydroxylamine hydrochloride $(0.1$ mole $)$ in $25 \mathrm{~mL}$ pyridine was refluxed for $2 \mathrm{hrs}$. On cooling the reaction mixture was poured over crushed ice and conc. HCl. The solid obtained was filtered, washed with water and crystallized from ethanol Scheme 1. Physical and analytical data of compounds are given in Table 1.

\section{Results and Discussion}

The structures of the synthesized compounds (4a-e) were confirmed on the basis of spectral and elemental analysis. The IR spectrum of 4a-e exhibited a band due to1650 $(\mathrm{C}=\mathrm{N}$, pyrazoline ring), $1590(\mathrm{C}=\mathrm{C}), 1150\left(-\mathrm{OCH}_{3}\right)$. Further, in their ${ }^{1} \mathrm{H}$ NMR (DMSO) spectrum, the appearance of a signal at $\delta 5.00-4.94\left(\mathrm{dd}, 1 \mathrm{H}, \mathrm{H}_{\mathrm{x}}\right.$ pyrazoline $), 3.51-3.44\left(\mathrm{dd}, 1 \mathrm{H}, \mathrm{H}_{\mathrm{B}}\right.$ pyrazoline) and $2.94-2.87\left(\mathrm{dd}, 1 \mathrm{H}, \mathrm{H}_{\mathrm{A}}\right.$ pyrazoline) confirms the presence of the pyrazoline ring. Similarly, the structures of compounds 5a-e were confirmed on the basis of spectral and elemental analysis. The IR spectrum of 5a-e exhibited a band due to $=\mathrm{CH}$ str. (3100$\left.3000 \mathrm{Cm}^{-1}\right), \mathrm{C}=\mathrm{C}$ str. $\left(1635-1495 \mathrm{Cm}^{-1}\right), \mathrm{C}-\mathrm{Cl}$ str. $\left(750-700 \mathrm{Cm}^{-1}\right), \mathrm{C}=\mathrm{N}$ (ring) $(1650-1580$ $\mathrm{Cm}^{-1}$ ) stretching vibration band which indicates the presence of the isoxazoline ring. Further, in their ${ }^{1} \mathrm{H}$ NMR (DMSO) spectrum, the appearance of a signal at $\delta 5.25-5.18\left(\mathrm{dd}, 1 \mathrm{H}, \mathrm{H}_{\mathrm{x}}\right.$ isoxazoline), 3.62-3.56 (dd, $1 \mathrm{H}, \mathrm{H}_{\mathrm{B}}$ isoxazoline) and $2.90-2.83\left(\mathrm{dd}, 1 \mathrm{H}, \mathrm{H}_{\mathrm{A}}\right.$ isoxazoline) confirms the presence of the isoxazoline ring.

The compounds 4 a-e and 5a-e were screened for their antibacterial activity against $E$ coli, Salmonella typhi, Staphylococcus aureus and Bacillus subtilis by using paper disc diffusion method $^{18,19}$ using Penicillin $(100 \mu \mathrm{b} / \mathrm{disc})$ as reference standard and antifungal activity against Aspergillus niger, Aspergillus flavus, Penicillium chrysogenum and Fusurium moneliforme by using Greseofulvin $(100 \mu \mathrm{b} / \mathrm{disc})$ as reference standard. The observed Minimum Inhibitory Concentrations (MIC) values for all the synthesized compounds are presented in Table 2 and 3. 
The investigation of antibacterial screening results indicate that compounds $\mathbf{4 b , c}$ and $\mathbf{5 b , c}$ shows promising activity and compounds $\mathbf{4 a}$ and $\mathbf{5 a}, \mathbf{e}$ poor activity against $E$. coli. Compounds $\mathbf{4 b}, \mathbf{c}$ and $\mathbf{5 c}, \mathbf{e}$ show good activity and compound $\mathbf{4 a , e}$ and $\mathbf{5 a}$ show low activity against Salmonella typhi. Compounds $\mathbf{4 b , c , d}$ and $\mathbf{5 b}, \mathbf{c}, \mathbf{d}$ show high activity and compounds 4a and 5a,e show low activity against Staphylococcus aureus. Compounds $\mathbf{4 b , c , e}$ and $\mathbf{5 b , c , d}$ show high activity and compounds $\mathbf{4 a , d}$ and $\mathbf{5 a}, \mathbf{e}$ show low activity against Bacillus subtilis. The investigation of antifungal activity data revealed that compounds $\mathbf{4 a , b , c , e}$ and $\mathbf{5 b}, \mathbf{c}, \mathbf{e}$ show inhibitory effect against $A$. niger and compounds $\mathbf{4 b , c}$ and $\mathbf{5 a , c , d}$ show inhibitory effect against $A$. flavus. Compounds $\mathbf{4 c , d}$ and 5a,b,c show inhibitory effect against $P$. chrysogenum, similarly compounds $\mathbf{4 c}$ show inhibitory effect against $F$. moneliforme. Remaining compounds are inactive against all the fungus. Results are shown in Table 2 and 3.

Table 2. Antibacterial screening results of the compounds (4a-e, 5a-e).

\begin{tabular}{ccccc}
\hline Compd & $\begin{array}{c}\text { E. } \\
\text { coli }\end{array}$ & $\begin{array}{c}\text { Salmonella } \\
\text { typhi }\end{array}$ & $\begin{array}{c}\text { Staphylococcus } \\
\text { aureus }\end{array}$ & $\begin{array}{c}\text { Bacillus } \\
\text { Subtilis }\end{array}$ \\
\hline $\mathbf{4 a}$ & 09 & 11 & 14 & 12 \\
$\mathbf{4 b}$ & 14 & 18 & 26 & 17 \\
$\mathbf{4 c}$ & 15 & 20 & 28 & 19 \\
$\mathbf{4 d}$ & 11 & 14 & 23 & 13 \\
$\mathbf{4 e}$ & 10 & 11 & 19 & 15 \\
$\mathbf{5 a}$ & 08 & 05 & 15 & 10 \\
$\mathbf{5 b}$ & 10 & 09 & 18 & 16 \\
$\mathbf{5 c}$ & 13 & 18 & 24 & 21 \\
$\mathbf{5 d}$ & 09 & 09 & 16 & 14 \\
$\mathbf{5 e}$ & 08 & 10 & 15 & 12 \\
Penicillin & 18 & 25 & 40 & 27 \\
DMSO & $-\mathrm{ve}$ & $-\mathrm{ve}$ & $-\mathrm{ve}$ & $-\mathrm{ve}$ \\
& \multicolumn{2}{c}{-ve no antibacterial activity }
\end{tabular}

Table 3. Antifungal screening results of the compounds (4a-e, 5a-e)

\begin{tabular}{ccccc}
\hline Compd & $\begin{array}{c}\text { Aspergillus } \\
\text { niger }\end{array}$ & $\begin{array}{c}\text { Aspergillus } \\
\text { flavus }\end{array}$ & $\begin{array}{c}\text { Penicillium } \\
\text { chrysogenum }\end{array}$ & $\begin{array}{c}\text { Fusarium } \\
\text { moneliforme }\end{array}$ \\
\hline $\mathbf{4 a}$ & $-\mathrm{ve}$ & $+\mathrm{ve}$ & $+\mathrm{ve}$ & $+\mathrm{ve}$ \\
$\mathbf{4 b}$ & $-\mathrm{ve}$ & $-\mathrm{ve}$ & $+\mathrm{ve}$ & $+\mathrm{ve}$ \\
$\mathbf{4 c}$ & $-\mathrm{ve}$ & $-\mathrm{ve}$ & $-\mathrm{ve}$ & $-\mathrm{ve}$ \\
$\mathbf{4 d}$ & $+\mathrm{ve}$ & $+\mathrm{ve}$ & $-\mathrm{ve}$ & $+\mathrm{ve}$ \\
$\mathbf{4 e}$ & $-\mathrm{ve}$ & $+\mathrm{ve}$ & $+\mathrm{ve}$ & $+\mathrm{ve}$ \\
$\mathbf{5 a}$ & $+\mathrm{ve}$ & $-\mathrm{ve}$ & $-\mathrm{ve}$ & $+\mathrm{ve}$ \\
$\mathbf{5 b}$ & $-\mathrm{ve}$ & $+\mathrm{ve}$ & $-\mathrm{ve}$ & $+\mathrm{ve}$ \\
$\mathbf{5 c}$ & $-\mathrm{ve}$ & $-\mathrm{ve}$ & $-\mathrm{ve}$ & $+\mathrm{ve}$ \\
$\mathbf{5 d}$ & $+\mathrm{ve}$ & $-\mathrm{ve}$ & $+\mathrm{ve}$ & $+\mathrm{ve}$ \\
$\mathbf{5 e}$ & $-\mathrm{ve}$ & $+\mathrm{ve}$ & $+\mathrm{ve}$ & $+\mathrm{ve}$ \\
Greseofulvin & $-\mathrm{ve}$ & $-\mathrm{ve}$ & $-\mathrm{ve}$ & $-\mathrm{ve}$ \\
Control- & $+\mathrm{ve}$ & $+\mathrm{ve}$ & $+\mathrm{ve}$ & $+\mathrm{ve}$ \\
$-\mathrm{ve}-$ No Growth : Antifungal activity,$+\mathrm{ve}-$ Growth : No Antifungal activity \\
\hline
\end{tabular}

\section{Spectral analysis of compounds}

1H-3-Phenyl-5-(6''-methoxynapthaline)-2-pyrazoline (4a)

${ }^{1} \mathrm{H}$ NMR DMSO: $\delta$ 7.79-7.68 (m, 4H, Ar-H), 7.56-7.53(m, 4H, Ar-H), 7.47-7.45 (d, 1H, J=8.8 $\mathrm{Hz}, \mathrm{Ar}-\mathrm{H}), 7.28$ (d, 1H, J=2.4 Hz, Ar-H), 7.14-7.12 (dd, 1H, J=2.4 Hz, Ar-H), 5.00-4.94 (dd, 1H, 
$\left.\mathrm{J}=10.8, \mathrm{H}_{\mathrm{x}}\right), 3.84\left(\mathrm{~s}, 3 \mathrm{H}, \mathrm{OCH}_{3}\right), 3.51-3.44\left(\mathrm{dd}, 1 \mathrm{H}, \mathrm{J}=10.8 \mathrm{~Hz}, \mathrm{H}_{\mathrm{B}}\right), 2.94-2.87(\mathrm{dd}, 1 \mathrm{H}, \mathrm{J}=10.8$ $\left.\mathrm{Hz}, \mathrm{H}_{\mathrm{A}}\right), \mathrm{IR}\left(\mathrm{KBr}\right.$ pellets $\left.\mathrm{Cm}^{-1}\right) 1650(\mathrm{C}=\mathrm{N}$, pyrazoline ring $), 1590(\mathrm{C}=\mathrm{C}), 1150\left(-\mathrm{OCH}_{3}\right)$.

1H-3-(4'-Bromophenyl)-5-(6''-methoxynapthaline)-2-pyrazoline (4b)

${ }^{1} \mathrm{H}$ NMR DMSO: $\delta$ 7.79-7.76 (m, 2H, Ar-H), 7.64-7.62(m, 2H, Ar-H), 7.49-7.46 (dd, 1H, J=1.6 $\mathrm{Hz}, \mathrm{Ar}-\mathrm{H}), 7.38-7.28$ (m, 4H, Ar-H), 7.14-7.12 (dd, 1H, J=2.8 Hz, Ar-H), 4.99-4.92 (dd, 1H, $\left.\mathrm{J}=12.0, \mathrm{H}_{\mathrm{x}}\right), 3.84\left(\mathrm{~s}, 3 \mathrm{H}, \mathrm{OCH}_{3}\right), 3.51-3.45\left(\mathrm{dd}, 1 \mathrm{H}, \mathrm{J}=11.2 \mathrm{~Hz}, \mathrm{H}_{\mathrm{B}}\right), 2.95-2.88(\mathrm{dd}, 1 \mathrm{H}, \mathrm{J}=10.8$ $\left.\mathrm{Hz}, \mathrm{H}_{\mathrm{A}}\right), \mathrm{IR}\left(\mathrm{KBr}\right.$ pellets $\left.\mathrm{Cm}^{-1}\right) 1645\left(\mathrm{C}=\mathrm{N}\right.$, pyrazoline ring), $1595(\mathrm{C}=\mathrm{C}), 1155\left(-\mathrm{OCH}_{3}\right)$.

1H-3-(4'-Flourophenyl)-5-(6',-methoxynapthaline)-2-pyrazoline (4c)

${ }^{1} \mathrm{H}$ NMR DMSO: $\delta$ 7.78-7.76 (m, 2H, Ar-H), 7.64-7.61(m, 2H, Ar-H), 7.49-7.46 (dd, 1H, J=1.6 $\mathrm{Hz}, \mathrm{Ar}-\mathrm{H}), 7.37-7.22$ (m, 4H, Ar-H), 7.14-7.13 (dd, 1H, J=2.0 Hz, Ar-H), 4.98-4.91 (dd, 1H, $\left.\mathrm{J}=11.8, \mathrm{H}_{\mathrm{x}}\right), 3.85\left(\mathrm{~s}, 3 \mathrm{H}, \mathrm{OCH}_{3}\right), 3.52-3.43\left(\mathrm{dd}, 1 \mathrm{H}, \mathrm{J}=11.6 \mathrm{~Hz}, \mathrm{H}_{\mathrm{B}}\right), 2.94-2.86(\mathrm{dd}, 1 \mathrm{H}, \mathrm{J}=11.0$ $\left.\mathrm{Hz}, \mathrm{H}_{\mathrm{A}}\right), \mathrm{IR}\left(\mathrm{KBr}\right.$ pellets $\left.\mathrm{Cm}^{-1}\right) 1640\left(\mathrm{C}=\mathrm{N}\right.$, pyrazoline ring), $1590(\mathrm{C}=\mathrm{C}), 1140\left(-\mathrm{OCH}_{3}\right)$.

1H-3-(4'-Methylphenyl)-5-(6' '-methoxynapthaline)-2-pyrazoline (4d)

${ }^{1} \mathrm{H}$ NMR DMSO: $\delta$ 7.73-7.68 (m, 3H, Ar-H), 7.60-7.58(d, 2H, J=8.4Hz, Ar-H), 7.47-7.44 (dd, $1 \mathrm{H}, \mathrm{J}=1.6 \mathrm{~Hz}, \operatorname{Ar}-\mathrm{H}), 7.20-7.11(\mathrm{~m}, 4 \mathrm{H}, \mathrm{Ar}-\mathrm{H}), 5.07-5.02\left(\mathrm{dd}, 1 \mathrm{H}, \mathrm{J}=8.8, \mathrm{H}_{\mathrm{x}}\right), 3.91(\mathrm{~s}, 3 \mathrm{H}$, $\left.\mathrm{OCH}_{3}\right), 3.55-3.48\left(\mathrm{dd}, 1 \mathrm{H}, \mathrm{J}=10.8 \mathrm{~Hz}, \mathrm{H}_{\mathrm{B}}\right), 3.15-3.08\left(\mathrm{dd}, 1 \mathrm{H}, \mathrm{J}=8.8 \mathrm{~Hz}, \mathrm{H}_{\mathrm{A}}\right), 2.37(\mathrm{~s}, 3 \mathrm{H}, \mathrm{Ar}-$ $\left.\mathrm{CH}_{3}\right)$, IR $\left(\mathrm{KBr}\right.$ pellets $\left.\mathrm{Cm}^{-1}\right) 1650\left(\mathrm{C}=\mathrm{N}\right.$, pyrazoline ring), $1590(\mathrm{C}=\mathrm{C}), 1150\left(-\mathrm{OCH}_{3}\right)$.

1H-3-(4'-Methoxyphenyl)-5-(6'”-methoxynapthaline)-2-pyrazoline (4e)

${ }^{1} \mathrm{H}$ NMR DMSO: $\delta$ 7.72-7.69 (m, 3H, Ar-H), 7.61-7.58(d, 2H, J=8.8, Ar-H), 7.47-7.44 (dd, $1 \mathrm{H}, \mathrm{J}=1.6 \mathrm{~Hz}, \mathrm{Ar}-\mathrm{H}), 7.22-7.10(\mathrm{~m}, 4 \mathrm{H}, \mathrm{Ar}-\mathrm{H}), 5.08-5.02\left(\mathrm{dd}, 1 \mathrm{H}, \mathrm{J}=8.4 \mathrm{~Hz}, \mathrm{H}_{\mathrm{x}}\right), 3.90(\mathrm{~s}$, $\left.6 \mathrm{H}, 2 \mathrm{XOCH}_{3}\right), 3.55-3.49\left(\mathrm{dd}, 1 \mathrm{H}, \mathrm{J}=9.8 \mathrm{~Hz}, \mathrm{H}_{\mathrm{B}}\right), 3.16-3.10\left(\mathrm{dd}, 1 \mathrm{H}, \mathrm{J}=9.2 \mathrm{~Hz}, \mathrm{H}_{\mathrm{A}}\right), \mathrm{IR}(\mathrm{KBr}$ pellets $\left.\mathrm{Cm}^{-1}\right) 1645\left(\mathrm{C}=\mathrm{N}\right.$ of pyrazoline ring), $1595(\mathrm{C}=\mathrm{C}), 1145\left(-\mathrm{OCH}_{3}\right)$

1H-3-Phenyl-5-(6',-methoxynapthaline)-2-isoxazoline (5a)

${ }^{1} \mathrm{H}$ NMR DMSO: $\delta$ 8.10-8.00 (m, 4H, Ar-H), 7.86-7.83(m, 4H, Ar-H), 7.77-7.75 (d, $1 \mathrm{H}$, $\mathrm{J}=8.6 \mathrm{~Hz}, \mathrm{Ar}-\mathrm{H}), 7.42(\mathrm{~d}, 1 \mathrm{H}, \mathrm{J}=2.8 \mathrm{~Hz}, \mathrm{Ar}-\mathrm{H}), 7.24-7.22(\mathrm{dd}, 1 \mathrm{H}, \mathrm{J}=2.6 \mathrm{~Hz}, \mathrm{Ar}-\mathrm{H}), 5.25-$ $5.18\left(\mathrm{dd}, 1 \mathrm{H}, \mathrm{J}=10.2, \mathrm{H}_{\mathrm{x}}\right), 3.86\left(\mathrm{~s}, 3 \mathrm{H}, \mathrm{OCH}_{3}\right), 3.62-3.56\left(\mathrm{dd}, 1 \mathrm{H}, \mathrm{J}=10.8 \mathrm{~Hz}, \mathrm{H}_{\mathrm{B}}\right), 2.90-2.83$ $\left(\mathrm{dd}, 1 \mathrm{H}, \mathrm{J}=10.8 \mathrm{~Hz}, \mathrm{H}_{\mathrm{A}}\right)$, IR ( $\mathrm{KBr}$ pellets $\left.\mathrm{Cm}^{-1}\right) 1612(\mathrm{C}=\mathrm{N}$ of isoxazoline ring), 1160 ($\left.\mathrm{OCH}_{3}\right), 1540(\mathrm{C}=\mathrm{C}),-\mathrm{C}-\mathrm{O}-\mathrm{N}$ (1231 of isoxazoline).

1H-3-(4'-Bromophenyl)-5-(6''-methoxynapthaline)-2-isoxazoline (5b)

${ }^{1} \mathrm{H}$ NMR DMSO: $\delta$ 8.11-8.02 (m, 2H, Ar-H), 7.92-7.90(m, 2H, Ar-H), 7.78-7.76 (dd, $1 \mathrm{H}$, $\mathrm{J}=1.8 \mathrm{~Hz}, \mathrm{Ar}-\mathrm{H}), 7.66-7.56(\mathrm{~m}, 4 \mathrm{H}, \mathrm{Ar}-\mathrm{H}), 7.39-7.37$ (dd, 1H, J=2.6 Hz, Ar-H), 5.20-5.16 $\left(\mathrm{dd}, 1 \mathrm{H}, \mathrm{J}=12.2, \mathrm{H}_{\mathrm{x}}\right), 3.88\left(\mathrm{~s}, 3 \mathrm{H}, \mathrm{OCH}_{3}\right), 3.55-3.50\left(\mathrm{dd}, 1 \mathrm{H}, \mathrm{J}=11.4 \mathrm{~Hz}, \mathrm{H}_{\mathrm{B}}\right), 2.96-2.89$ (dd, $\left.1 \mathrm{H}, \mathrm{J}=10.8 \mathrm{~Hz}, \mathrm{H}_{\mathrm{A}}\right)$, ), IR ( $\mathrm{KBr}$ pellets $\left.\mathrm{Cm}^{-1}\right) 1605\left(\mathrm{C}=\mathrm{N}\right.$ of isoxazoline ring), $1165\left(-\mathrm{OCH}_{3}\right)$, $1535(\mathrm{C}=\mathrm{C}),-\mathrm{C}-\mathrm{O}-\mathrm{N}$ (1225 of isoxazoline).

1H-3-(4'-Flourophenyl)-5-(6' '-methoxynapthaline)-2-isoxazoline (5c)

${ }^{1} \mathrm{H}$ NMR DMSO: $\delta$ 8.12-8.04 (m, 2H, Ar-H), 7.91-7.89(m, 2H, Ar-H), 7.77-7.75 (d, $1 \mathrm{H}$, $\mathrm{J}=1.8 \mathrm{~Hz}, \mathrm{Ar}-\mathrm{H}), 7.62-7.52$ (m, 4H, Ar-H), 7.38-7.36 (dd, 1H, J=2.2 Hz, Ar-H), 5.19-5.15 $\left(\mathrm{dd}, 1 \mathrm{H}, \mathrm{J}=12.2, \mathrm{H}_{\mathrm{x}}\right), 3.86\left(\mathrm{~s}, 3 \mathrm{H}, \mathrm{OCH}_{3}\right), 3.52-3.46\left(\mathrm{dd}, 1 \mathrm{H}, \mathrm{J}=11.0 \mathrm{~Hz}, \mathrm{H}_{\mathrm{B}}\right), 2.94-2.86(\mathrm{dd}$, $\left.1 \mathrm{H}, \mathrm{J}=11.0 \mathrm{~Hz}, \mathrm{H}_{\mathrm{A}}\right)$, ), IR $\left(\mathrm{KBr}\right.$ pellets $\left.\mathrm{Cm}^{-1}\right) 1615\left(\mathrm{C}=\mathrm{N}\right.$ of isoxazoline ring), $1165\left(-\mathrm{OCH}_{3}\right)$, $1540(\mathrm{C}=\mathrm{C}),-\mathrm{C}-\mathrm{O}-\mathrm{N}$ (1231 of isoxazoline).

1H-3-(4'-Methylphenyl)-5-(6''-methoxynapthaline)-2-isoxazoline (5d)

${ }^{1} \mathrm{H}$ NMR DMSO: $\delta$ 8.00-7.95 (m, 3H, Ar-H), 7.90-7.88(d, 2H, J=8.6 Hz, Ar-H), 7.80-7.77 (dd, 1H, J=1.6 Hz, Ar-H), 7.61-7.54 (m, 4H, Ar-H), 5.15-5.10 (dd, 1H, J=8.8, $\mathrm{H}_{\mathrm{x}}$ ), 3.89 (s, $\left.3 \mathrm{H}, \mathrm{OCH}_{3}\right), 3.55-3.48\left(\mathrm{dd}, 1 \mathrm{H}, \mathrm{J}=8.0 \mathrm{~Hz}, \mathrm{H}_{\mathrm{B}}\right), 2.99-2.90\left(\mathrm{dd}, 1 \mathrm{H}, \mathrm{J}=8.8 \mathrm{~Hz}, \mathrm{H}_{\mathrm{A}}\right), 2.33(\mathrm{~s}, 3 \mathrm{H}$, 
$\left.\mathrm{Ar}-\mathrm{CH}_{3}\right)$, ), IR ( $\mathrm{KBr}$ pellets $\left.\mathrm{Cm}^{-1}\right) 1610\left(\mathrm{C}=\mathrm{N}\right.$ of isoxazoline ring), $1155\left(-\mathrm{OCH}_{3}\right), 1545$ $(\mathrm{C}=\mathrm{C}),-\mathrm{C}-\mathrm{O}-\mathrm{N}$ (1240 of isoxazoline).

1H-3-(4'-Methoxyphenyl)-5-(6' '-methoxynapthaline)-2-isoxazoline (5e)

${ }^{1} \mathrm{H}$ NMR DMSO: $\delta$ 8.11-8.04 (m, 3H, Ar-H), 7.91-7.88(d, 2H, J=8.6, Ar-H), 7.81-7.78 (dd, 1H, $\mathrm{J}=1.8 \mathrm{~Hz}, \operatorname{Ar}-\mathrm{H}), 7.59-7.47(\mathrm{~m}, 4 \mathrm{H}, \mathrm{Ar}-\mathrm{H}), 5.12-5.08\left(\mathrm{dd}, 1 \mathrm{H}, \mathrm{J}=8.6 \mathrm{~Hz}, \mathrm{H}_{\mathrm{x}}\right), 3.91(\mathrm{~s}, 6 \mathrm{H}$, $\left.2 \mathrm{XOCH}_{3}\right), 3.55-3.49\left(\mathrm{dd}, 1 \mathrm{H}, \mathrm{J}=9.2 \mathrm{~Hz}, \mathrm{H}_{\mathrm{B}}\right), 2.96-2.90\left(\mathrm{dd}, 1 \mathrm{H}, \mathrm{J}=9.2 \mathrm{~Hz}, \mathrm{H}_{\mathrm{A}}\right)$, ), IR (KBr pellets $\left.\mathrm{Cm}^{-1}\right) 1615(\mathrm{C}=\mathrm{N}$ of isoxazoline ring $), 1150\left(-\mathrm{OCH}_{3}\right), 1530(\mathrm{C}=\mathrm{C}),-\mathrm{C}-\mathrm{O}-\mathrm{N}(1220$ of isoxazoline $)$.

\section{Conclusion}

The synthesized 2-pyrazolines 4a-e and 2-isoxazoline 5a-e all are novel. Compounds with electron releasing groups such as methoxy and compounds having pharmacophores such as chloro, fluoro, bromo groups and both these groups are present in one moiety exhibited best antimicrobial activity. The data reported in this article may be helpful guide for the medicinal chemist who is working in this area.

\section{Acknowledgement}

The authors are thankful to Mr. A. R. Muley, Miss K R Bagul dept. of chemistry, Miss V.G. Ambhore Dept. of Biotechnology Milind College of science Aurangabad for help in antimicrobial study. The authors are also thankful to the Principal Dr. B.C. Ghoble for providing facilities.

\section{References}

1 Kargaokar S S, Patil P H, Shah M T and Parekh H H, Indian J Pharm Sci., 1996, 58, 222.

2 Palaskar E, Aytemir M, Uzbay I T and Erol D, Eur J Med Chem., 2001, 36, 539-543.

3 Bilgin A A, Palaskar E and Sunal R, Aezneim Forsch Drug Res., 1993, 43, 1041.

4 Ozdemir Z, Kandilici H B, Gumusel B, Calis U and Bilgin A A, Eur J Med Chem., 2007, 42, 373-379.

5 Ruhoglus O, Ozdemir Z, Calis U, Gumusel B and Bilgin A A, Aezneim Forsch Drug Res., 2005, 55, 431-436.

6 Udupi R H, Kushnoor A R and Bhat A R, Indian J Hetrocyclic Chem., 1998, 8, 63.

7 Taylor E C and Patel H H, Tetrahedron, 1992, 48, 8089-8100.

8 Regaila H A, El-Bayanki A K and Hammad M, Egypt J Chem., 1979, 20, 197.

9 Krishna R B, Panade R, Bhaithwal S P and Parmar S S, Eur Med J Chem., 1980, 15567.

10 Husain M J and Shukla S, Indian J Chem., 1986, 25B, 983.

11 Mizabuchis and Satoy, Agri Biol Chem., 1984, 48, 2771.

12 Bhakunin D S and Chaturvedi R, J Nat Prod., 1984, 47, 585.

13 Vittorio F, Ronsisvalle G, Pappalardo M S and Blandino G, Chem Abstr., 1985, 103, 19721c.

14 Lapage F and Hublot B, Chem Abstr., 1996, 113, 211964g.

15 Shivkumar B and Nargund L V G, Indian J Hetrocyclic Chem., 1998, 8, 27.

16 Simmonds M S, Blaney W M, Monuche F D and Marini Bettollo, J Chem Ecol., 1996, 16, 365.

17 Nagano M, Sakai J, Mizukai M, Nakamura N, Misaka E, Kobayashi S and Tomita K, Ipn Kokai, JP54073774, 1979, 774; Chem Abstr., 1980, 92, 41922v.

18 Gillespie S H, Medical Microbiology Illustrated, (Butterworth Heinemann Ltd., Oxford, United Kingdom), 1994, 234.

19 Hawkey P M and Lewis D A, Medical Bacteriology- A Practical Approach, (Oxford University Press, Oxford, United Kingdom), 1994, 181. 


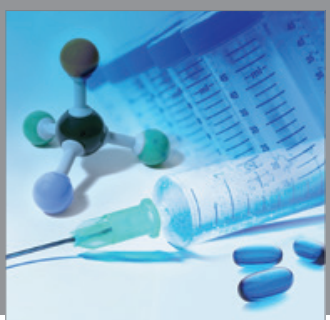

International Journal of

Medicinal Chemistry

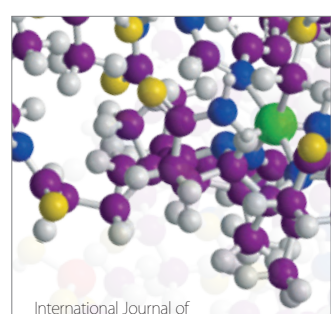

Carbohydrate Chemistry

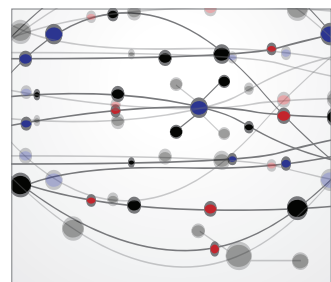

The Scientific World Journal
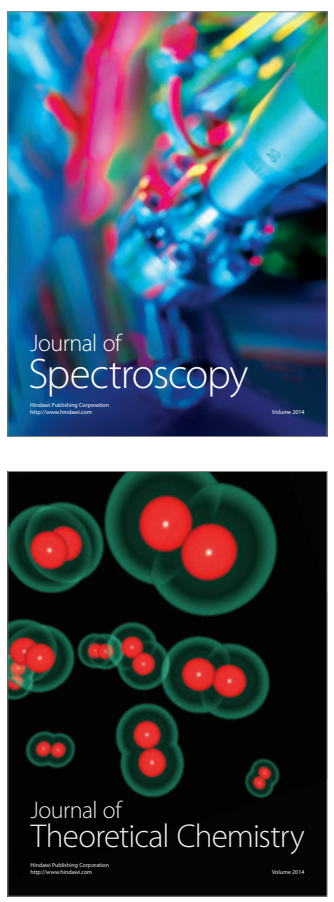
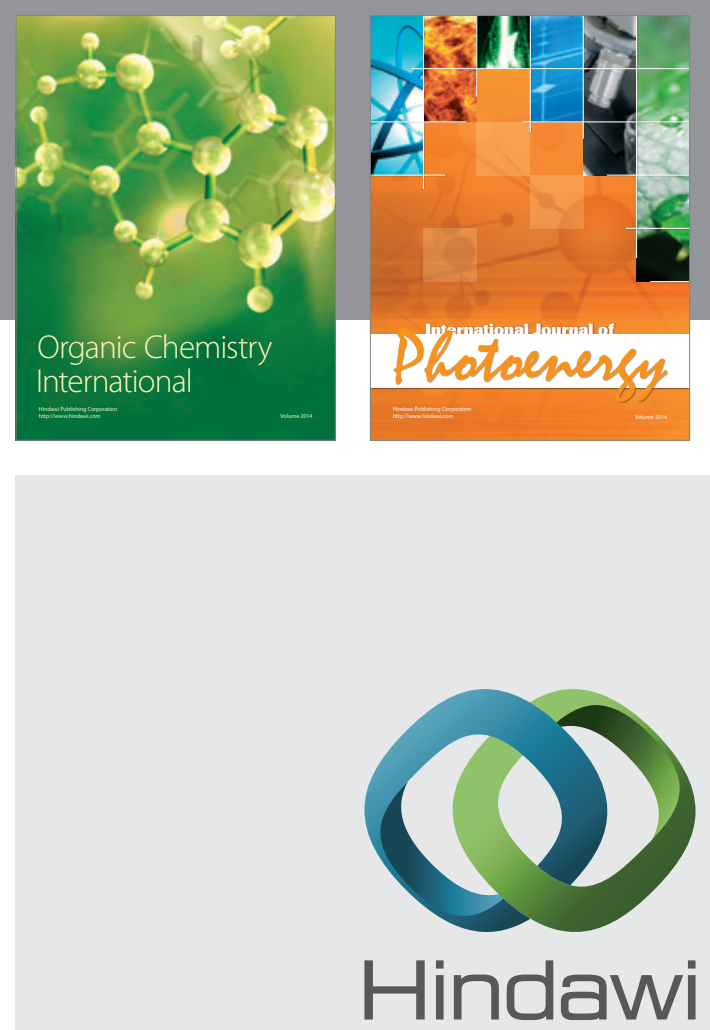

Submit your manuscripts at

http://www.hindawi.com
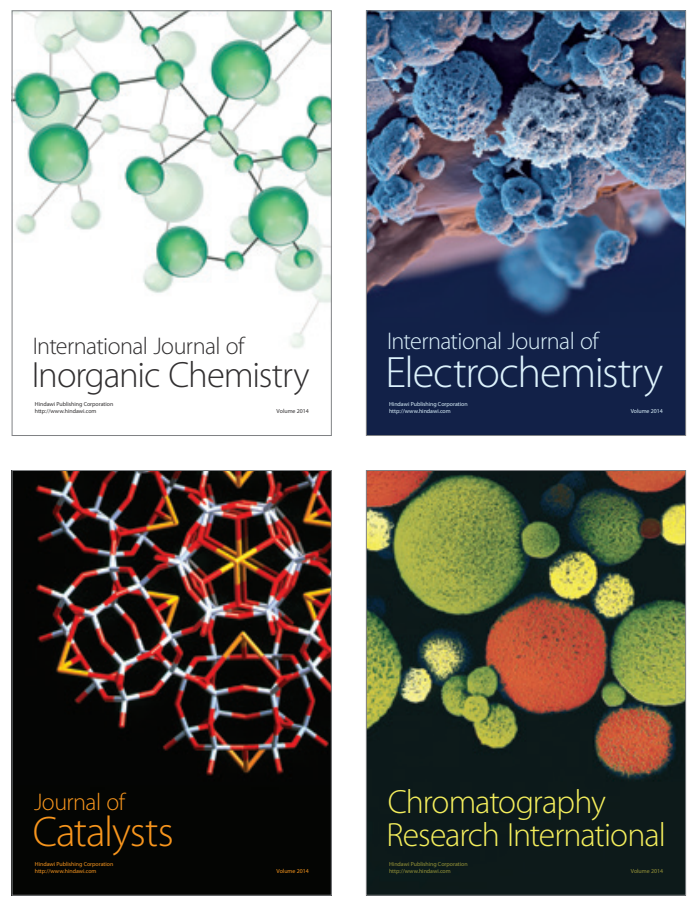
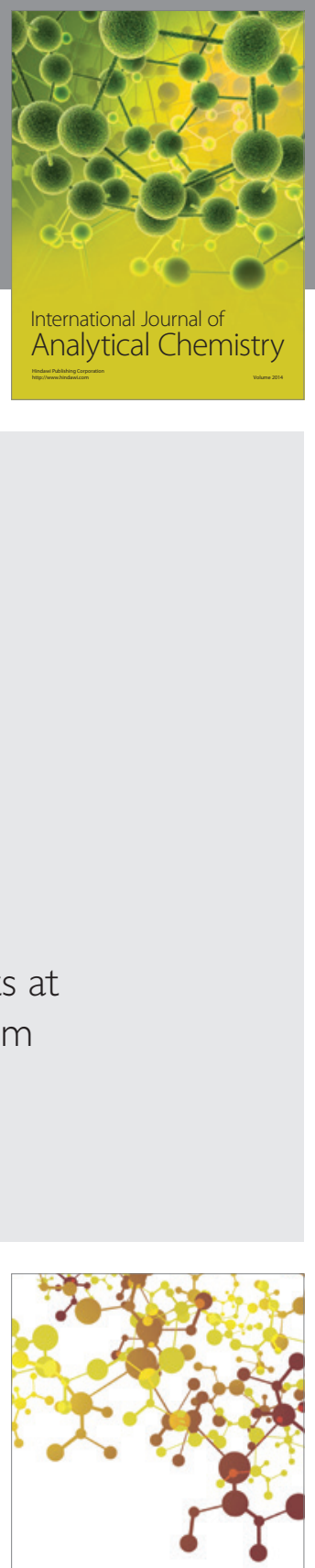

Journal of

Applied Chemistry
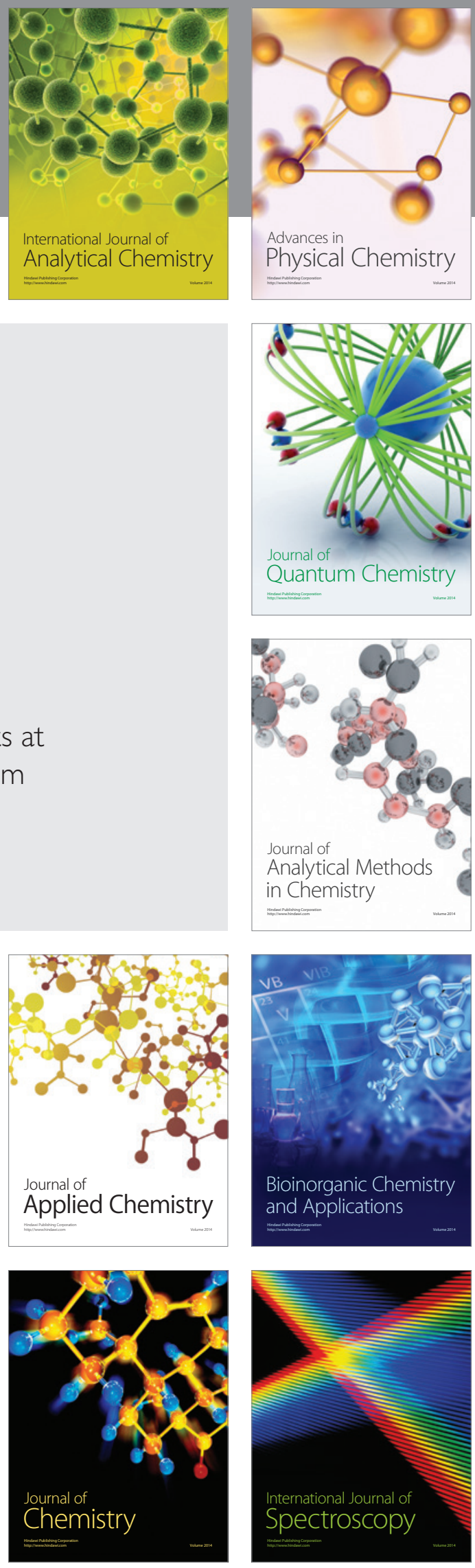